\title{
Dynamic regimes of fluids simulated by multiparticle-collision dynamics
}

\author{
M. Ripoll, K. Mussawisade, R. G. Winkler, and G. Gompper \\ Institut für Festkörperforschung, Forschungszentrum Jülich, D-52425 Jülich, Germany
}

(Received 3 February 2005; published 5 July 2005)

\begin{abstract}
We investigate the hydrodynamic properties of a fluid simulated with a mesoscopic solvent model. Two distinct regimes are identified, the "particle regime" in which the dynamics is gaslike and the "collective regime" where the dynamics is fluidlike. This behavior can be characterized by the Schmidt number, which measures the ratio between viscous and diffusive transport. Analytical expressions for the tracer diffusion coefficient, which have been derived on the basis of a molecular-chaos assumption, are found to describe the simulation data very well in the particle regime, but important deviations are found in the collective regime. These deviations are due to hydrodynamic correlations. The model is then extended in order to investigate self-diffusion in colloidal dispersions. We study first the transport properties of heavy pointlike particles in the mesoscopic solvent, as a function of their mass and number density. Second, we introduce excluded-volume interactions among the colloidal particles and determine the dependence of the diffusion coefficient on the colloidal volume fraction for different solvent mean-free paths. In the collective regime, the results are found to be in good agreement with previous theoretical predictions based on Stokes hydrodynamics and the Smoluchowski equation.
\end{abstract}

DOI: 10.1103/PhysRevE.72.016701

PACS number(s): 47.11.+j, 82.20.Wt, 82.70.-y

\section{INTRODUCTION}

The dynamics of complex fluids such as colloidal suspensions, dilute or semidilute polymer solutions, biological macromolecules, membranes, and aqueous surfactant solutions, is often governed by the hydrodynamic behavior of the solvent. Due to a large separation of length and time scales between the atomic scale of the solvent molecules and the mesoscopic scale of the solute, direct simulation approaches with explicit atomistic solvent are prohibitively costly in computer time. Therefore, several mesoscale simulation techniques have been developed in recent years in order to bridge the length- and time-scale gap. In particular, latticegas automata (LGA) [1,2], lattice Boltzmann (LB) [3-5], smoothed-particle hydrodynamics (SPH) [6,7], dissipative particle dynamics (DPD) [8-10], direct simulation Monte Carlo (DSMC) [11,12], fluid particle dynamics [13], and others, have been investigated. The basic idea of all these approaches is very similar: To obtain hydrodynamic behavior on length scales much larger than the atomic scale, the detailed interactions and dynamics of the solvent molecules are not important; instead mass and momentum conservation are the essential ingredients to obtain the correct hydrodynamic behavior. Therefore, the dynamics on the microscopic scale can be strongly simplified, as long as the conservation laws are strictly satisfied. The different methods listed above differ in the way the solvent dynamics is implemented.

Two main classes of mesoscopic simulation techniques can be distinguished, which are lattice and off-lattice methods. Lattice gas and lattice Boltzmann methods fall into the first class, while direct simulation Monte Carlo, dissipative particle dynamics, and fluid particle dynamics fall into the second class. Off-lattice approaches have the advantage that Galilean invariance is typically satisfied. Moreover, the interaction of the off-lattice solvent with solutes such as colloids, polymers, and membranes can be taken into account more naturally.
The mesoscale simulation technique, which we are investigating in this paper, was introduced by Malevanets and Kapral [14] a few years ago. It is a variant of the DSMC method, in which binary collisions are replaced by multiparticle collisions in a prescribed collision volume. This method has been called multiparticle-collision dynamics (MPCD) or stochastic rotation dynamics (SRD). It employs a discretetime dynamics with continuous velocities and local multiparticle collisions. Mass and momentum are conserved quantities and it has been demonstrated that the hydrodynamic equations are satisfied $[14,15]$.

Certain transport coefficients, in particular the viscosity, of this solvent model have been studied intensively. Analytical expressions have been derived from kinetic theory by generalizing pointlike collisions to finite collision volumes [16-19]. The theoretical expressions describe numerical results very well.

In this article, we study the transport coefficients as a function of the parameters of the MPCD fluid, in particular the mean free path in units of the size of the collision volume. We find two distinct regimes, in which the dynamics is either gaslike or fluidlike. This behavior can be characterized by the Schmidt number, which measures the ratio between viscous and diffusive transport. We find that MPCD allows us to tune the fluid behavior such that large Schmidt numbers are obtained and momentum transport dominates over mass transport. Analytical expressions [17-19] for the tracer diffusion coefficient, which have been derived on the basis of a molecular-chaos assumption, are found to describe the simulation data very well for large mean free paths, but fail in the fluid regime. The reason is a build-up of correlations among the fluid particles by hydrodynamic interactions, which leads to enhanced diffusion coefficients. We will show that the latter leads to nonexponentially decaying velocityautocorrelation functions at small mean free paths. Independent of the mean free path, we find that the algorithm reproduces the algebraic long-time decay typical in fluids. 
In a further step, we investigate the diffusion of a heavy tracer particle in a MPCD solvent. It is very important to understand the contribution of the solvent dynamics on the solute diffusion. Two limiting situations are found: either Brownian or hydrodynamic behavior, depending on the collision time and the rotation angle. We explore the range of parameters where these different dynamical behaviors appear, and show how they emerge from the mesoscopic dynamics.

Finally, we study self-diffusion in colloidal dispersions with excluded-volume interactions as a function of the volume fraction. To this end, the MPCD method is combined with molecular dynamic simulations. We find that such a hybrid model displays the proper dynamics for the same parameter regime where the hydrodynamic behavior is found for the fluid. Our results in the collective regime are in good agreement with previous theoretical predictions based on Stokes hydrodynamics and the Smoluchowski equation [20].

\section{THE MODEL}

The fluid is modeled by $N$ point particles, which are determined by their positions $\mathbf{r}_{i}$ and velocities $\mathbf{v}_{i}$, with $i$ $=1, \ldots, N$. Positions and velocities are continuous variables, which evolve in discrete increments of time. The mass $m$ associated with the particles is taken to be the same, but more generally, different masses can be assigned. The algorithm consists of two steps, streaming and collision. In the streaming step the particles move ballistically according to their velocities during a time increment $h$, to which we will refer as collision time. Thereby, the evolution rule is

$$
\mathbf{r}_{i}(t+h)=\mathbf{r}_{i}(t)+h \mathbf{v}_{i}(t) .
$$

In the collision step, the particles are sorted into collision boxes, and interact with all other particles in the same collision box. The collision boxes are typically the unit cells of a $d$-dimensional cubic lattice with lattice constant $a$, although other geometries would be possible. The collision is then defined as a rotation of the velocities of all particles in a box in a comoving frame with its center of mass. Thus, the velocity of the $i$ th particle after the collision is

$$
\mathbf{v}_{i}(t+h)=\mathbf{v}_{\mathrm{c} . \mathrm{m} ., i}(t)+\mathcal{R}(\alpha)\left[\mathbf{v}_{i}(t)-\mathbf{v}_{\mathrm{c} . \mathrm{m} . i}(t)\right],
$$

where $\mathcal{R}(\alpha)$ is a stochastic rotation matrix and $\mathbf{v}_{\text {c.m. } . i}(t)$ $=\Sigma_{j}^{(i, t)}\left(m \mathbf{v}_{j}\right) / \Sigma_{j} m$ is the velocity of the center of mass of all particles $j$, which are located in the collision box of particle $i$ at time $t$. The conservation of local momentum and kinetic energy is guaranteed by construction. In two dimensions, the rotation of the relative velocity is simply given by an angle $\pm \alpha$. Here $\alpha$ is a parameter of the model; the sign is chosen randomly for each cell. In three dimensions, various schemes for the random collisions are possible $[14,18,21]$. The one employed in this paper consist in choosing a random direction in space for each box around which the relative velocities are rotated by an angle $\alpha$. A detailed explanation of the implementation is given in Ref. [21].

In order to ensure Galilean invariance for the full range of parameters, a random shift of the collision grid has to be
TABLE I. Summary of relevant parameters for the simple fluid with the MPCD model.

\section{Parameters}

$a$ : Collision box size

$m$ : Mass of the fluid particle

$T$ : Temperature

$h$ : Collision time

$\alpha$ : Rotation angle

$L$ : Linear system size

$N$ : Total number of particles

$\varrho$ : Mass density, $\varrho=N m / L^{d}$

$\Lambda$ : Mean free path, $\Lambda=h \sqrt{k_{B} T / m}$

Dimensionless quantities

$\gamma$ : Decorrelation factor, $\gamma=(2 / 3)(1-\cos \alpha)(\rho-1) / \rho$

$\rho$ : Particles per cell, $\rho=\varrho a^{d} / m=N(a / L)^{d}$

$\lambda$ : Scaled mean free path, $\lambda=\Lambda / a$

performed in the execution of the collision step [16,22]. As a consequence of such a shift, the collision environment of each particle is independent of the average local velocity, and no special reference frame exists. Random shifts also facilitate the transfer of momentum between neighboring particles.

In the simulations, $N$ particles are initially placed at random in a cubic system of linear extension $L$. The average number of particles in a collision box is $\rho=N(a / L)^{d}$, the scaled number density. Starting from an arbitrary distribution of velocities, only a few steps are required to reach the Maxwell Boltzmann velocity distribution. The equilibrium temperature $T$ is then given by the average kinetic energy $m\left\langle\mathbf{v}_{i}^{2}\right\rangle=3 k_{B} T$, where $k_{B}$ is the Boltzmann constant. In the simulations, we scale length and time according to $\hat{x}=x / a$ and $\hat{t}=t \sqrt{k_{B} T / m a^{2}}$, which corresponds to the choice $m=1$, $a=1$, and $k_{B} T=1$ of reference units. The scaled mean free path is then given by $\lambda=\hat{h}$. Basic parameters and the definitions of dimensionless quantities are collected in Table I.

\section{DYNAMICAL PROPERTIES}

The kinematic viscosity $\nu=\eta / \varrho$ has been calculated theoretically $[14-19,22,23]$ by means of kinetic theory and its validity has been checked with simulations. The total kinematic viscosity, $\nu=\nu_{\text {kin }}+\nu_{\text {coll }}$, is the sum of two contributions, the kinetic viscosity $\nu_{\text {kin }}$ and the collisional viscosity $\nu_{\text {coll }}$, which have been calculated in two and three dimensions. In three dimensions, the expressions $[18,19]$

$$
\begin{gathered}
\frac{\nu_{\text {coll }}}{\sqrt{k_{B} T a^{2} / m}}=\frac{1}{\lambda} \frac{(1-\cos \alpha)}{18}\left(1-\frac{1}{\rho}\right), \\
\frac{\nu_{\text {kin }}}{\sqrt{k_{B} T a^{2} / m}}=\lambda\left[\frac{1}{(4-2 \cos \alpha-2 \cos 2 \alpha)} \frac{5 \rho}{\rho-1}-\frac{1}{2}\right]
\end{gathered}
$$

have been derived. 

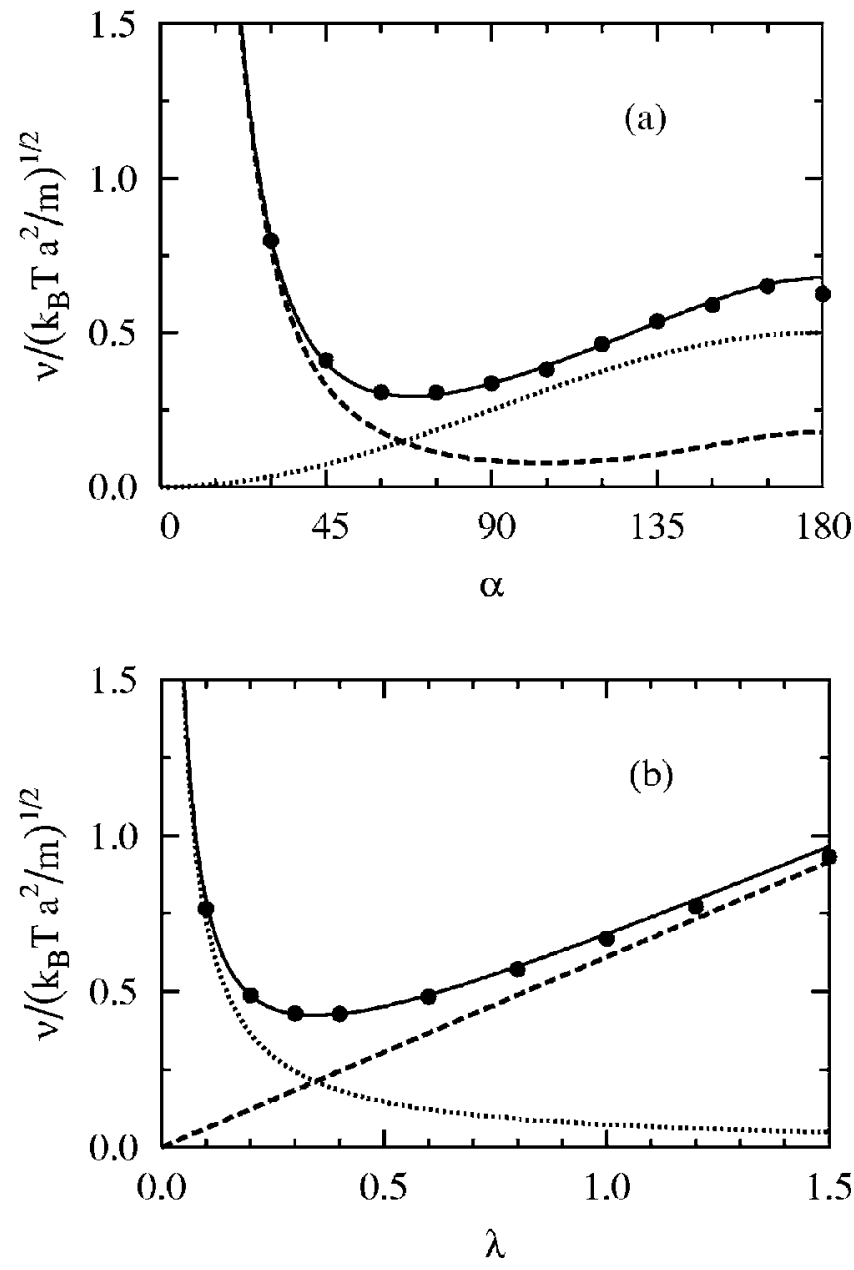

FIG. 1. Dimensionless kinematic viscosity for the simple fluid in MPCD. The symbols are the simulation results, the solid line is the total theoretical prediction, the dotted line is the collisional contribution, and the dashed line the kinetic contribution. In both cases the system size is $L / a=20$. (a) $\alpha$ dependence with $\lambda=0.2$ and $\rho$ $=10$. (b) $\lambda$ dependence with $\alpha=130$ and $\rho=5$.

The total kinematic viscosity has been determined numerically by the procedure explained in Ref. [24]. Briefly, a three-dimensional system is considered with periodic boundary conditions in two dimensions and planar walls in the third dimension. Stick boundary conditions at the walls are implemented by considering bounce-back collisions with the walls. A gravitational field is applied in one direction parallel to the walls. After a relaxation time, the system reaches a stationary state with a parabolic velocity profile between the walls and in the direction of the force. This is Poiseuille flow. It is known [25] that the measured maximum velocity of the parabola is inversely proportional to the viscosity of the fluid. The viscosity data obtained in this way are presented in Fig. 1 together with the theoretical predictions of Eq. (3). The obtained agreement is quite remarkable, in contrast to the case of other mesoscopic simulation techniques such as dissipative particle dynamics [26]. Density fluctuations can also be included in the theory [19], which noticeably improves the agreement with the simulations results for small number densities; for $\rho=5$ and $\rho=10$, these contributions are

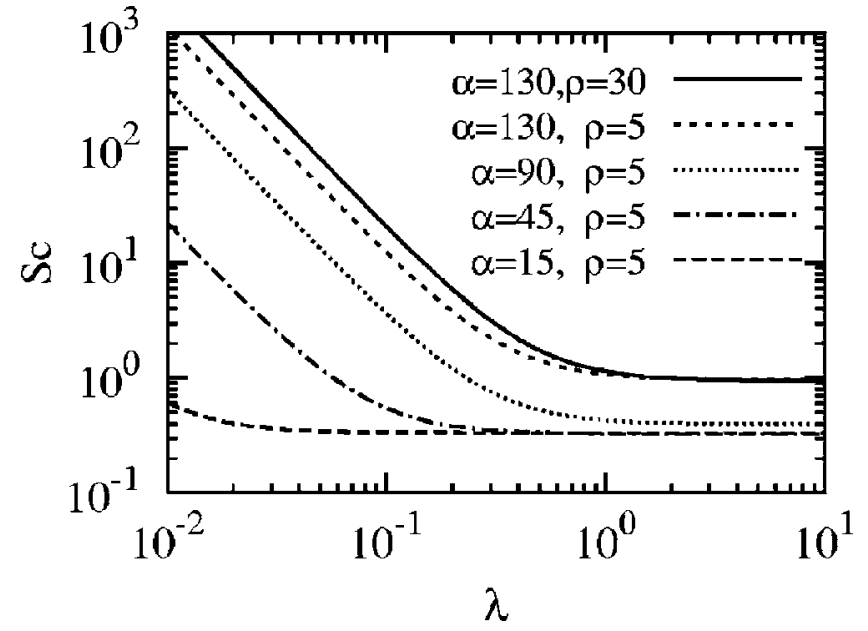

FIG. 2. Theoretical Schmidt number versus collision time. The $\alpha$ and $\rho$ parameters are specified in the plot.

negligible. Alternative methods to determine the viscosity from simulations have been employed in Refs. [19,17], where a system under shear flow and vorticity correlations have been used, respectively.

The ratio between the kinetic and the collisional contributions to the kinematic viscosity varies considerably with the model parameters, as can be seen easily from the theoretical expressions (3). In Fig. 1 the total kinematic viscosity and its two contributions are plotted as a function of the rotation angle and the collision time step. The collisional contribution is dominant for large collision angles and small collision times, while the kinetic viscosity dominates in the opposite case of small collision angles and large collision times.

Kinetic transport is due to the movement of the particles themselves, i.e., when a particle moves it carries a certain amount of the relevant quantities as momentum and energy, while collisional transport is due to transfer of energy and momentum from one particle to another during collisions. In MPCD, kinetic transport is therefore dominant when the mean free path is larger than the size of the collision box and for small values of the rotation angle. If the rotation angle is small, there is little exchange of momentum between particles due to collisions. The situation where the kinetic transport dominates is characteristic for gases. In fluids the usual situation is the opposite, the transport of momentum is mainly due to collisions.

A convenient measure of the importance of hydrodynamics is the Schmidt number $\mathrm{Sc}=\nu / D$, where $\nu$ is the kinematic viscosity and $D$ the diffusion coefficient. Thus, Sc is the ratio between momentum transport and mass transport. It is known that this number for gases is smaller than but on the order of unity, while in fluids like water it is on the order of $10^{2}$ to $10^{3}$. A prediction for the Schmidt number of a MPCD fluid can be obtained from the theoretical expressions (3) for the kinematic viscosity, and the diffusion coefficient, see Eq. (17) below. In Fig. 2, we plot the theoretical prediction for Sc as a function of the collision time for different values of the rotation angle. This shows that Sc becomes considerably larger than unity for the same range of parameters where the collisional viscosity is considerably larger than the kinetic 
viscosity (Fig. 1). We will show that the dynamical behavior in the two limits is fundamentally different. We will call the parameter region of large rotation angles and small collision times the "collective regime" and the opposite region the "particle regime". This classification has similar consequences as the one introduced in dissipative particle dynamics (DPD) [27], although we do not investigate wavelength dependent properties here.

\section{SIMPLE FLUID CORRELATIONS}

Correlations between particles are responsible for hydrodynamic interactions. Therefore, we are interested in characterizing the velocity correlations in a MPCD fluid.

\section{A. Velocity autocorrelation functions}

An analytical expression for the velocity autocorrelation function (VACF) has been derived in Refs. [17,18]. The collision step in Eq. (2) can be rewritten as

$$
\begin{aligned}
\mathbf{v}_{i}(n h)= & \mathbf{v}_{i}((n-1) h)+(\mathcal{R}(\alpha)-I) \\
& \times\left[\mathbf{v}_{i}((n-1) h)-\mathbf{v}_{\mathrm{c} . \mathrm{m} ., i}((n-1) h)\right],
\end{aligned}
$$

where $I$ is the unit matrix and $t=n h$ is the discretized time, with $n$ the number of collision steps. By multiplying this expression with the velocity at time zero and taking thermal averages, we obtain

$$
\begin{aligned}
\left\langle\mathbf{v}_{i}(n h) \mathbf{v}_{i}(0)\right\rangle= & \left(1-\gamma_{\alpha}\right)\left\langle\mathbf{v}_{i}((n-1) h) \mathbf{v}_{i}(0)\right\rangle \\
& +\gamma_{\alpha}\left\langle\mathbf{v}_{\mathrm{c} . \mathrm{m} . i}((n-1) h) \mathbf{v}_{i}(0)\right\rangle .
\end{aligned}
$$

Here, the rotational average over an arbitrary vector $\mathbf{A}$ in three dimensions is obtained from geometrical arguments to be

$$
\langle(\mathcal{R}(\alpha)-\mathbb{I}) \mathbf{A}\rangle=-\frac{2}{3}(1-\cos \alpha)\langle\mathbf{A}\rangle \equiv-\gamma_{\alpha}\langle\mathbf{A}\rangle .
$$

This particular value of $\gamma_{\alpha}$ arises from the implementation of the rotation chosen in this paper. The remaining problem is to calculate the last term in Eq. (5). First, we neglect density fluctuations in the average of the center of mass velocity, which yields $\left\langle\mathbf{v}_{\mathrm{c} . \mathrm{m}, i}(n h)\right\rangle \simeq\left\langle\Sigma_{j}^{(i, n)} \mathbf{v}_{j}\right\rangle / \rho$. Furthermore, a molecular-chaos assumption implies that

$$
\left\langle\mathbf{v}_{\text {c.m. } . i}((n-1) h) \mathbf{v}_{i}(0)\right\rangle \simeq \frac{1}{\rho}\left\langle\mathbf{v}_{i}((n-1) h) \mathbf{v}_{i}(0)\right\rangle .
$$

This approximation means that of all the particles in the collision box of particle $i$ after $(n-1)$ collisions, only particle $i$ itself makes a nonzero contribution to the correlation function. This is the same as assuming that none of the other particles has any information about the state of particle $i$ at any time. The correlation at a certain time step can then be expressed in terms of the previous time step as

$$
\left\langle\mathbf{v}_{i}(n h) \mathbf{v}_{i}(0)\right\rangle \simeq\left(1-\frac{\rho-1}{\rho} \gamma_{\alpha}\right)\left\langle\mathbf{v}_{i}((n-1) h) \mathbf{v}_{i}(0)\right\rangle .
$$

This implies that in this approximation, the VACF shows an exponential decay

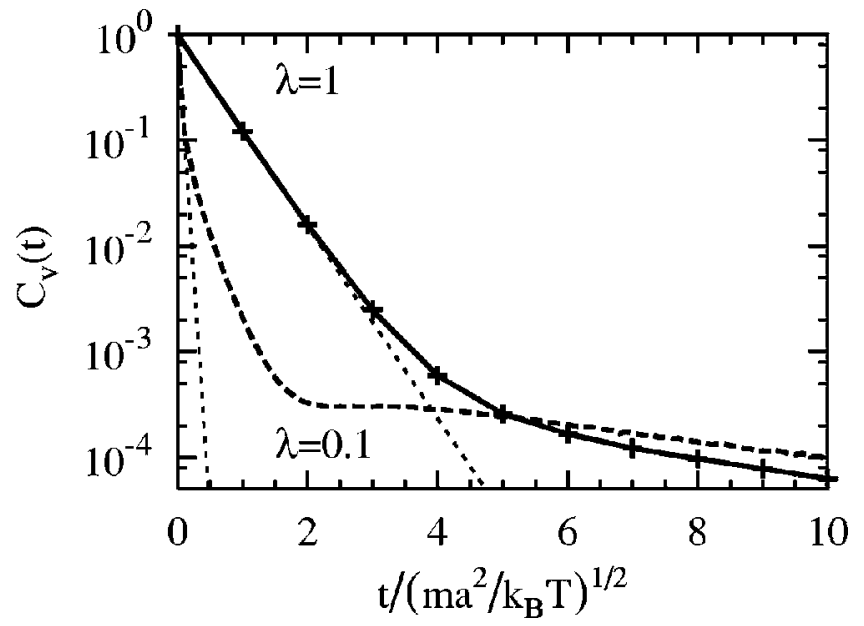

FIG. 3. Normalized velocity autocorrelation function as a function of the dimensionless time for mean free paths $\lambda=1$ and $\lambda$ $=0.1$. Dashed lines correspond to the exponential decays in Eq. (9). In both cases the number density is $\rho=5$, the rotation angle $\alpha$ $=130$, and the system size $L / a=20$.

$$
C_{v}(n h) \equiv \frac{\left\langle\mathbf{v}_{i}(n h) \mathbf{v}_{i}(0)\right\rangle}{\left\langle v_{i}^{2}(0)\right\rangle} \simeq(1-\gamma)^{n}
$$

where the normalization factor follows from the equipartition theorem $\left\langle\mathbf{v}_{i}^{2}(0)\right\rangle=3 k_{B} T / m$. The decorrelation factor $\gamma$ is defined as

$$
\gamma=\frac{2}{3}(1-\cos \alpha)\left(1-\frac{1}{\rho}\right) \equiv \gamma_{\alpha} \gamma_{\rho} .
$$

From Eq. (9), a characteristic time $\tau_{0}=-h / \ln (1-\gamma)$ can be extracted. Up to this time, the VACF follows the exponential decay for every set of parameters. However, the collective phenomena responsible for the hydrodynamic behavior appear at much later times.

In Fig. 3, simulation results of the VACF are presented for two different mean free paths $\lambda$. The theoretical prediction (9) is also displayed for both values of $\lambda$. For $\lambda=1$ the exponential decay is followed with very good accuracy until the crossover to the long-time tail behavior occurs. For $\lambda$ $=0.1$ the purely exponential decay is followed only in the first collision; for long times, a long-time-tail behavior is observed similarly as for $\lambda=1$. What is different in this case is that after the first collision the system enters an intermediate regime where the VACF decay is significantly slower than the one described by the molecular-chaos approximation but is not yet the algebraic tail. Note that for the investigated rotation angle of $\alpha=130$, the mean free path $\lambda=1$ corresponds to the particle regime, while $\lambda=0.1$ corresponds to the collective regime.

It is interesting to note that for short times, the VACF decays monotonically only in the case that the correlation parameter $\gamma$ is smaller than unity. If $\gamma \geqslant 1$, Eq. (9) predicts that the VACF exhibits damped oscillations. We have checked that this oscillatory behavior is indeed observed in the simulations. However, the viscosity curves show no par- 


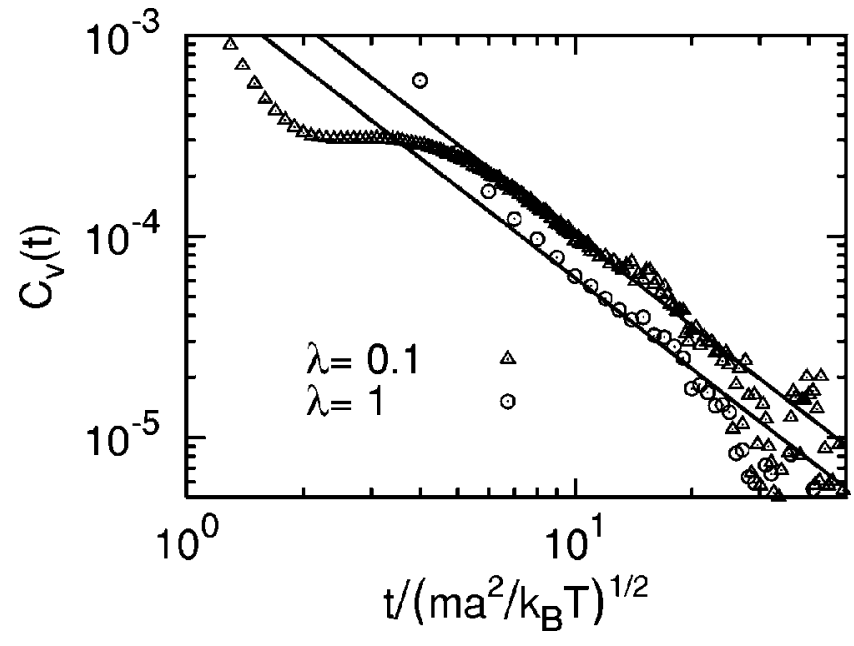

FIG. 4. Time dependence of the normalized velocity autocorrelation function. The parameters are the same as in Fig. 3. The data are compared with long-time tail prediction $t^{-1 / 3}$. The amplitude predicted in Eq. (11) is (within the statistical error) exact for $\lambda=1$ and about $10 \%$ larger for $\lambda=0.1$.

ticular features when this happens (compare Fig. 1, where the VACF for $\rho=10$ becomes oscillatory for $\alpha \geqslant 132$ ).

\section{B. Long-time tails}

It is well known [28-30] that the long-time behavior of the VACF in $d$-dimensional fluids in thermal equilibrium shows a universal behavior. This corresponds to a power-law tail, for which the explicit form can be calculated from a mode-coupling theory as [29]

$$
C_{v}(t) \simeq\left(\frac{d-1}{d \rho}\right) \frac{1}{[4 \pi(D+\nu) t]^{d / 2}},
$$

where $\nu$ and $D$ are the transport coefficients of the fluid.

The results obtained for the long-time behavior of the VACF are consistent with the general prediction for fluids in thermal equilibrium in Eq. (11). The algebraic power $t^{-3 / 2}$ is clearly reproduced in our simulations as can be seen in Fig. 4. The value of the amplitude in Eq. (11) is related to the kinematic viscosity $\nu$ and the diffusion coefficient $D$. Since both values are known for the MPCD fluid and discussed in this paper, quantitative comparison can also be performed. We find that the value for $\lambda=1$ is exactly reproduced by our simulations within the accuracy of the results, while the amplitude obtained for $\lambda=0.1$ is about $10 \%$ smaller than the theoretic prediction. Ihle and Kroll [16] obtain good agreement in a two-dimensional MPCD fluid with the expected $t^{-1}$ behavior over a comparable time window.

The effect of finite system size can be seen in Fig. 4 for times $\hat{t} \geqq 20$, where the VACF crosses over from the algebraic to a faster, exponential decay. This effect is similar to that observed for the time dependence of the temperature autocorrelation function for a random-solid dissipativeparticle-dynamics system [31]. There, it can be proved that the correlations decay faster after a time, where hydrodynamic modes become relevant which are truncated by the system size.

\section{Importance of many-body correlations}

In the previous section, an exponential decay of the VACF has been theoretically predicted. This behavior is a consequence of the approximation in Eq. (7) which neglects any correlation among the particles in the same collision box at all times. In order to improve Eq. (7), we have to go beyond the molecular-chaos approximation. This is a formidable task. We start the procedure by calculating the center-ofmass correlation average for the first collision and, consecutively, the second and so on. For $n=1$ the approximation in Eq. (7) is exact $\left\langle\mathbf{v}_{\text {c.m. } . i}(0) \mathbf{v}_{i}(0)\right\rangle=\left\langle\mathbf{v}_{i}^{2}(0)\right\rangle / \rho$. This is the reason why for the first time step, $C_{v}(h)$ agrees perfectly in all simulations. For $n=2$ it reads

$$
\begin{aligned}
\left\langle\mathbf{v}_{\mathrm{c} . \mathrm{m} ., i}(h) \mathbf{v}_{i}(0)\right\rangle= & \frac{1}{\rho} \sum_{j}^{(i, 1)}\left\langle\left\{\mathbf{v}_{j}(0)+(\mathcal{R}(\alpha)-\mathbb{I})\right.\right. \\
& \left.\left.\times\left[\mathbf{v}_{j}(0)-\mathbf{v}_{\mathrm{c} . \mathrm{m} . j}(0)\right]\right\} \mathbf{v}_{i}(0)\right\rangle \\
= & \frac{1}{\rho}\left(1-\gamma_{\alpha}\right)\left\langle v_{i}^{2}(0)\right\rangle+\frac{\gamma_{\alpha}}{\rho^{2}} \sum_{j} \sum_{k}^{(i, 1)}\left\langle\mathbf{v}_{k}(0) \mathbf{v}_{i}(0)\right\rangle \\
& \equiv\left(\frac{1-\gamma_{\alpha}}{\rho}+\frac{\gamma_{\alpha}}{\rho^{2}} \zeta_{1}\right)\left\langle v_{i}^{2}(0)\right\rangle
\end{aligned}
$$

where $\zeta_{1}$ denotes the number of particles that are neighbors of particle $i$ at both times $t=h$ and $t=0$. We use the term "neighbors" for particles within the same collision box. The approximation in Eq. (7) is recovered for $\zeta_{1}=1$. This is the case when only the actual particle is considered to be in both collision boxes. As we have seen above, this is not a good approximation in the collective regime.

We denote the average number of remaining neighbors that one particle is revisiting after $n$ collisions as $\zeta_{n}$. This number could in principle be calculated analytically by probabilistic arguments, but in order to get a flavor of the improvement that such numbers produce in the theory, we determine $\zeta_{n}$ numerically in our simulations. As expected, these numbers strongly depend on the system parameters. A detailed study has not been performed, but we have observed that the number of remaining neighbors seems to be a universal function of the root-mean-square displacement of the tagged particle.

The measured numbers $\zeta_{n}$ are presented in Fig. 5 as a function of the root-mean-square displacement $\langle(\mathbf{r}(t)$ $\left.-\mathbf{r}(0))^{2}\right\rangle^{1 / 2}=\sqrt{6 D t}$, where $D$ is the diffusion coefficient and $t=n h$. The diffusion coefficient is the one obtained from the analytical expression which will be deduced in the next section [see Eq. (17)]. The data for different mean free paths seem to fall onto a single master curve with reasonable accuracy. When the numerical values of $D$ (discussed in the next section) are used instead of the theoretical result, the data collapse becomes even more accurate. For the large mean free path $\lambda=1$, the first collision takes place when $\sqrt{6 D t} / a \simeq 2$, which implies that $\zeta_{1} \simeq 1$ is a good approximation. Note that in the representation chosen in Fig. 5, $\zeta_{n} \simeq 1$ corresponds to the abscissa. The same displacement for a small mean free path $\lambda=0.1$ takes place when the particle has been involved in 80 collisions on average. The first collision 


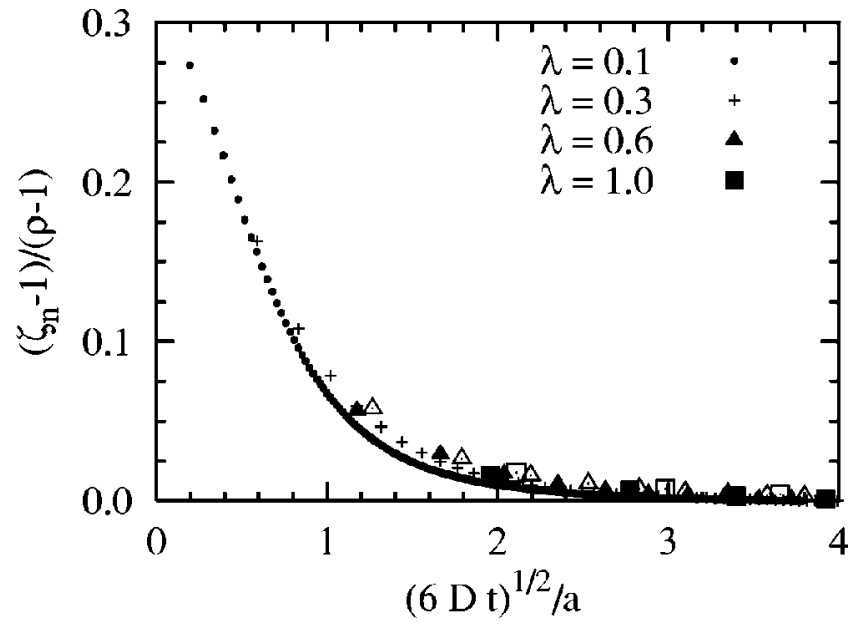

FIG. 5. Number of remaining neighbors in a collision box after $n$ collisions as a function of the root-mean-square displacement. Solid symbols correspond to $\rho=5, \alpha=130$ with mean free path specified in the legend. Open symbols correspond to $\rho=10, \alpha$ $=110$ with $\lambda=0.6(\triangle)$ and $\lambda=1(\square)$.

for $\lambda=0.1$ takes place when the average displacement is much smaller and many of the particles are still in the same collision box, which makes $\zeta_{1} \simeq 1$ a bad approximation. Indeed, we can infer from Fig. 5 that $\zeta_{1} \simeq 2.1$ for $\lambda=0.1$ and $\rho=5$, and $\zeta_{1} \simeq 3.5$ for $\lambda=0.1$ and $\rho=10$.

Following the same procedure as employed in Eq. (12), the velocity correlation function can be calculated for $n=3$,

$$
\begin{aligned}
\left\langle\mathbf{v}_{\mathrm{c} . \mathrm{m} ., i}(2 h) \mathbf{v}_{i}(0)\right\rangle= & \frac{\left\langle v_{i}^{2}(0)\right\rangle}{\rho}\left[(1-\gamma)\left(1-\gamma+\frac{\gamma}{\rho} \zeta_{2}\right)\right. \\
& \left.+\frac{\gamma}{\rho}(1-\gamma) \zeta_{1}+\frac{\gamma^{2}}{\rho}\left(\zeta_{2}+\delta \zeta_{2}\right)\right],
\end{aligned}
$$

where $\delta \zeta_{2}$ is determined by

$$
\zeta_{2}+\delta \zeta_{2} \equiv \frac{1}{\left\langle v_{i}^{2}(0)\right\rangle} \sum_{j}^{(i, 2)} \sum_{l}^{(j, 1)} \sum_{k}^{(l, 0)}\left\langle\mathbf{v}_{k}(0) \mathbf{v}_{i}(0)\right\rangle
$$

This is the number of neighbors of particle $i$ at the two times $t=2 h$ and $t=0$ together with the neighbors of the neighbors, or the result of ring collisions. Let us consider two particles $i$ and $k$, which are in the same collision box at $t=2 h$ but not at $t=0$. If one, $k$, has been neighbor of a third particle $j$ at $t=h$ and this $j$ was neighbor of $i$ at $t=0$, then this combination also contributes to the correlation function. To obtain a reasonable prediction for this number is obviously not trivial. Furthermore, this relation will become more interconnected and difficult to predict for further time steps. It can be checked that with the approximations $\zeta_{n}=1$ and $\delta \zeta_{n}=0$, Eq. (13) reduces to Eq. (7), and consequently the exponential decay in Eq. (9) is recovered.

Now we come back to the correlation average in Eq. (5) which can be expanded with the help of Eq. (4) - without any approximation-

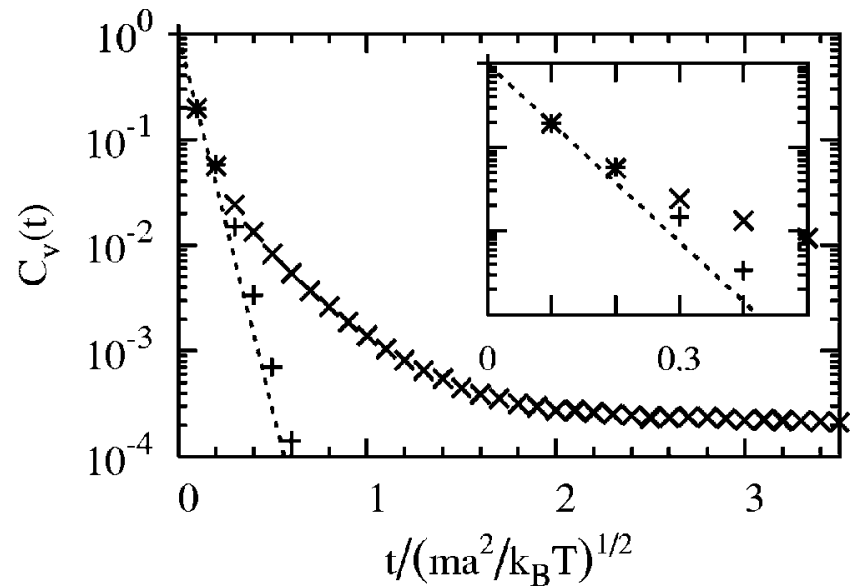

FIG. 6. Time dependence of the normalized velocity autocorrelation function. The dashed line is the exponential decay in Eq. (9), crosses $(\times)$ are the simulation results and pluses $(+)$ are the predicted values obtained by employing the $\zeta_{n}$ numbers, as indicated in Eq. (15). The parameters of the simulation are $\rho=10, \alpha=110, \lambda$ $=0.1$, and $L / a=20$. The inset is a zoom into the regime of the first few collisions.

$$
\begin{aligned}
\left\langle\mathbf{v}_{i}(n h) \mathbf{v}_{i}(0)\right\rangle= & \left\langle v_{i}^{2}(0)\right\rangle(1-\gamma)^{n} \\
& -\gamma \sum_{k=1}^{n}(1-\gamma)^{n-k}\left\langle\mathbf{v}_{\mathrm{c} . \mathrm{m} ., i}((k-1) h) \mathbf{v}_{i}(0)\right\rangle
\end{aligned}
$$

The predictions for short times can be improved compared to Eq. (9) by employing the results of Eqs. (12) and (13) on the right-hand side of Eq. (15), but setting $\zeta_{n} \simeq 1$ and $\delta \zeta_{n} \simeq 0$ for $n \geqslant 3$ as before. The result is shown in Fig. 6. We observe that the prediction for the second collision $C_{v}(2 h)$ now agrees perfectly with the simulation data, which confirms our arguments. Nevertheless, the prediction for further steps is still only a small improvement compared to the exponential decay in Eq. (9).

The most relevant conclusion at this point is that in the collective regime the MPCD algorithm accounts for manybody collisions which are crucial for the build-up of correlations. This is known to be the origin of the hydrodynamic behavior in fluids.

\section{SELF-DIFFUSION}

We study now the consequences of the different behavior in the two hydrodynamic regimes, which have been introduced in Sec. II, on the self-diffusion coefficient.

\section{A. Diffusion coefficient}

In the Green-Kubo formalism, the self-diffusion coefficient is given by $D=\frac{1}{3} \int_{0}^{\infty} d t\langle\mathbf{v}(t) \mathbf{v}(0)\rangle$. In the case that the time is discretized the integral has to be replaced by $[17,22]$ 


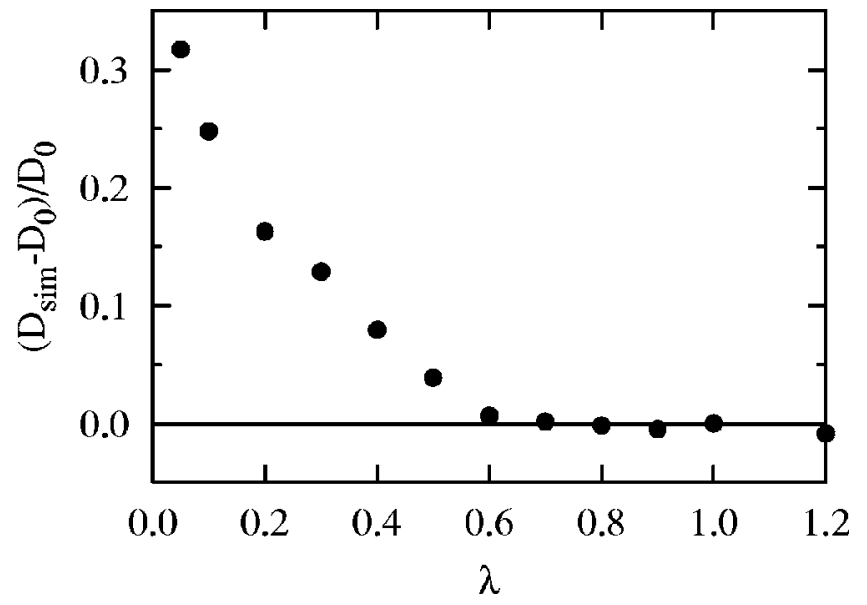

FIG. 7. Relative deviation $\Delta D=\left(D_{\text {sim }}-D_{0}\right) / D_{0}$ of the simulated diffusion coefficient from the Brownian approximation, as a function of the scaled mean free path $\lambda$. Full circles are simulation results, the solid line represents the analytical expression of $D_{0}$ in Eq. (17). Simulation parameters are $\alpha=130, \rho=5$, and $L / a=20$.

$$
D=\frac{1}{3}\left[\frac{1}{2}\left\langle v^{2}(0)\right\rangle h+\sum_{n=1}^{\infty}\langle\mathbf{v}(n h) \mathbf{v}(0)\rangle\right] h .
$$

In order to obtain an analytical prediction for the diffusion coefficient, an expression for $\langle\mathbf{v}(n h) \mathbf{v}(0)\rangle$ is required. The Brownian approximation for the VACF given by Eq. (9) yields

$$
D_{0}=\frac{k_{B} T}{m} h\left(\frac{1}{\gamma}-\frac{1}{2}\right)
$$

with $\gamma$ defined in Eq. (10). This expression coincides with that of Ref. [17] with a different notation.

In the simulations, the diffusion coefficient is determined by a linear fit of the mean-square displacement for long times. We have checked that equivalent results for $D$ are also obtained directly from the VACF by employing Eq. (16).

Figure 7 shows the relative deviation $\Delta D=\left(D_{\text {sim }}\right.$ $\left.-D_{0}\right) / D_{0}$ of the diffusion coefficient from the expression (17). This expression should be a good approximation as long as the exponential decay (9) of the VACF applies. This is indeed the case for $\lambda>0.6$, which means that the longtime tail for these values has a negligible contribution for the diffusion coefficient. This is reasonable since the deviation from the exponential behavior appears when the VACF has decayed typically by three orders of magnitude ( see $\lambda=1.0$ in Fig. 3). In contrast, Fig. 7 shows that the deviation from the Brownian behavior (17) increases with decreasing $\lambda$ for $\lambda$ $<0.5$. This can be understood from the VACF since for small $\lambda$ the deviation from the exponential decay appears much earlier. Figure 3 shows that for $\lambda=0.1$ the VACF has decayed only by about one order of magnitude when the deviation starts. This translates into a noticeable increment of the diffusion coefficient. This difference can be understood as a hydrodynamic enhancement of the diffusion coefficient for large values of the Schmidt number.
The diffusion coefficient for a simple MPCD fluid in two dimensions has been determined by Ihle and Kroll [17]. In their Fig. 15, results for $\lambda=0.113$ are presented as a function of the rotation angle; deviations from the theoretical prediction are found for large values of $\alpha$, which is in the range of parameters which we identify as the collective regime. They arrive at a similar conclusion that this is due to multiple encounters among particles. In three dimensions, some numerical results of the diffusion coefficient have been presented in Ref. [32], and good agreement with the molecularchaos approximation has been found for a large range of number densities. However, the employed parameters (which correspond to $\lambda>0.5)$ all belong to the particle regime, where we argue that a good agreement with the theory should be expected.

At this stage we come back to the discussion in Sec. III about the Schmidt number $\mathrm{Sc}=\nu / D$. The analytic expression can be calculated from the viscosity $\nu$ in Eq. (3) and the diffusion coefficient in Eq. (17), as was already pointed out in Refs. $[17,33,34]$. Note that $\mathrm{Sc}$ increases rapidly for small values $\lambda \ll 1$ of the mean free path, where $\mathrm{Sc} \sim h^{-2}$. This allows arbitrary large values of the Schmidt number. Although very small values of the collision time significantly reduce the efficiency of the simulations, there is a range of $\lambda$ values which are not too small but still display fluid behavior corresponding to high Sc. On the other hand, the hydrodynamic enhancement of the diffusion coefficient in the collective regime leads to values of Sc which are smaller than predicted by the analytical approximation. By substituting the numerically determined diffusion coefficient, it can be checked that Sc is indeed smaller, but still large enough to display a fluidlike behavior.

\section{B. Continuum time limit}

It is interesting to discuss the limit of small collision times $h \rightarrow 0$, and small rotation angles $\alpha \rightarrow 0$. The leading contributions in the theoretical expressions (3) of the kinetic and collisional viscosity read in this limit

$$
\nu_{\mathrm{coll}} \simeq \frac{m \gamma_{\rho}}{36 a}\left(\frac{\alpha^{2}}{h}\right), \quad \nu_{\mathrm{kin}} \simeq \frac{k_{B} T}{a^{3} \gamma_{\rho}}\left(\frac{h}{\alpha^{2}}\right)
$$

with $\gamma_{\rho}$ defined in Eq. (10). This result shows that a finite viscosity is obtained in the continuum limit only if the ratio $\alpha^{2} / h$ is kept constant. The additive term due to discrete times in Eq. (17) naturally vanishes in the continuum limit, because $\gamma \sim \alpha^{2}$.

The expressions (18) for the kinetic and collisional contributions to the viscosity show that the collective regime, where $\nu_{\text {coll }} \gg \nu_{\text {kin }}$, corresponds to $\alpha^{2} / h \gg 1$ in the continuum limit. In this regime, the leading contribution to the diffusion coefficient (17) is found to be

$$
D \simeq \frac{3 k_{B} T}{\gamma_{\rho}}\left(\frac{h}{\alpha^{2}}\right)
$$

The related Schmidt number 


$$
\mathrm{Sc}=\frac{\nu}{D} \simeq \frac{1}{108} \frac{m^{2} \gamma_{\rho}^{2}}{a k_{B} T}\left(\frac{\alpha^{2}}{h}\right)^{2}
$$

can be very large since $\alpha^{2} / h \gg 1$. This shows that the model has a proper continuum limit. However, due to the requirement of very small collision times, this limit is not very convenient from a computational point of view.

It is very satisfactory to see that the Stokes-Einstein relation is satisfied in this case, since the diffusion coefficient is inversely proportional to the viscosity

$$
D \simeq \frac{k_{B} T}{6 \pi \rho \nu_{\mathrm{coll}} R} \text { with } R=\frac{2 a}{\pi \rho}
$$

and defines an effective particle radius inversely proportional to the number density. We want to emphasize, however, that the Stokes-Einstein relation is not only satisfied in the continuum limit, but always when the additive term 1/2 in Eq. (17) can be neglected and the collisional dominates the kinetic viscosity. In this case, Eq. (21) is also valid.

\section{DYNAMICS OF EMBEDDED PARTICLES}

After the behavior of a simple MPCD fluid has been characterized, the next important question is how complex fluids can be modeled. As first step, we investigate the behavior of a single heavy pointlike particle, which could represent a solute particle or a colloidal sphere embedded in a simple fluid. Also, the monomers in a polymer chain can be represented as point particles [34-37]. This is a quite convenient strategy, since the solute-solvent interactions are modeled by just including the pointlike solute particles in the collision step. Then we study different concentrations of these heavy particles.

\section{A. Single heavy tracer particle}

For the simulation of heavy pointlike particles embedded in a solvent, the algorithm is the same as described for the simple fluid in Sec. II. The only point where the higher mass plays a role is in the calculation of the velocity of the center of mass, where the different particle masses have to be taken into account via $\mathbf{v}_{\mathrm{c} . \mathrm{m} ., i}(t)=\Sigma_{j}^{(i, t)}\left(m_{j} \mathbf{v}_{j}\right) / \Sigma_{j} m_{j}$. In thermal equilibrium, the average kinetic energy of light and heavy particles is the same. Therefore, the average momentum of the heavy particle of mass $M$ is a factor $(\mathrm{M} / \mathrm{m})^{1 / 2}$ larger than the average momentum of a light particle. This implies that a heavy particle has a larger contribution in the center-of-mass velocity than a light particle. Since the center-of-mass velocity and therefore also the velocities of all particles after the collision step depends on $M$ and the mass $m \rho$ of the solvent particles in a collision cell, the effective coupling between the solvent and the solute must depend in general on the ratio $M /(m \rho)$.

We denote the heavy particle position and velocity with capital letters $\mathbf{R}$ and $\mathbf{V}$. Of course, all types of particles are involved in the center-of-mass calculation or other sums over particles. The VACF can be calculated in the molecularchaos approximation as explained in Sec. IV A, except for

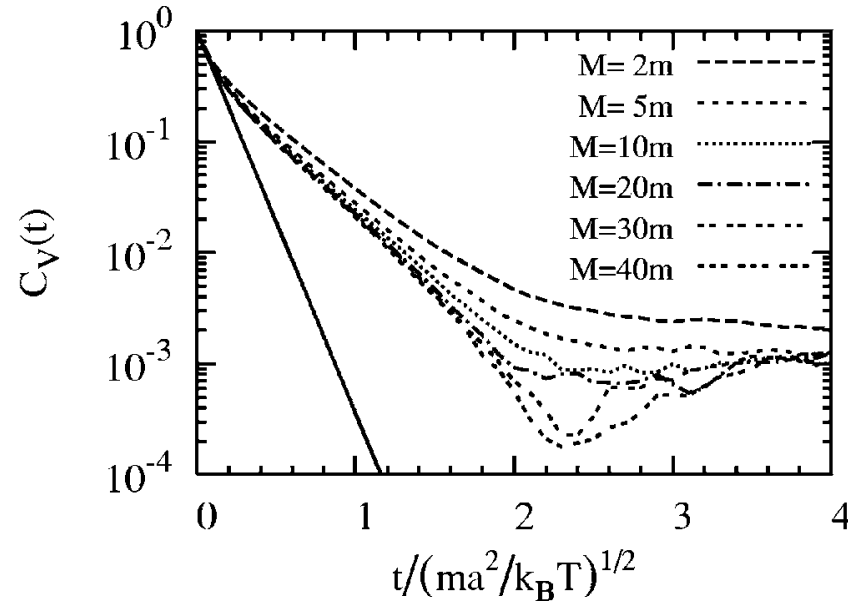

FIG. 8. Time dependence of the normalized velocity autocorrelation function for different heavy particles. Simulation parameters are $\lambda=0.1, \alpha=130, L / a=20$, and $\rho=M / m$. Dashed lines are simulation results and the solid line is the molecular-chaos approximation (24).

the center-of-mass correlation in Eq. (7), which for the heavy particle yields

$$
\left\langle\mathbf{V}_{\mathrm{c} . \mathrm{m} .}((n-1) h) \mathbf{V}(0)\right\rangle \simeq \frac{M}{m \rho+M}\langle\mathbf{V}((n-1) h) \mathbf{V}(0)\rangle
$$

because in the collision box of the heavy particle the total mass is $(M+m \rho)$. The correlation at time zero depends now on the heavy particle mass

$$
\left\langle\mathbf{V}^{2}(0)\right\rangle=3 \frac{k_{B} T}{M} .
$$

By inserting these results in the expression equivalent to Eq. (5), we obtain the molecular-chaos approximation for the normalized VACF of the heavy particle

$$
C_{V}(t) \equiv \frac{\langle\mathbf{V}(n h) \mathbf{V}(0)\rangle}{\left\langle V^{2}(0)\right\rangle} \simeq(1-\gamma)^{n},
$$

where the decorrelation factor $\gamma$ is now given by

$$
\gamma=\gamma_{\alpha} \frac{m \rho}{m \rho+M} \equiv \gamma_{\alpha} \gamma_{1}
$$

and $\gamma_{1}$ is defined for one heavy particle in the presence of $\rho$ fluid particles, in contrast to $\gamma_{\rho}$ in Eq. (10), where a fluid particle is surrounded by $(\rho-1)$ other fluid particles.

In Fig. 8 results for the normalized VACF of one heavy particle in the collective regime are presented for different values of its mass. The solvent mass density has been chosen to be equal to the solute mass, i.e., $\rho=M / m$. In this way, $\gamma$ $=\gamma_{\alpha} / 2$ and the analytical expression (24) is independent of the heavy particle mass. Figure 8 shows that after the second collision all the simulation data exhibit a nonexponential decay. This is not very surprising, since a similar behavior was observed for the simple fluid in Fig. 3 for parameter values within the collective regime. A slightly slower decay is dis- 


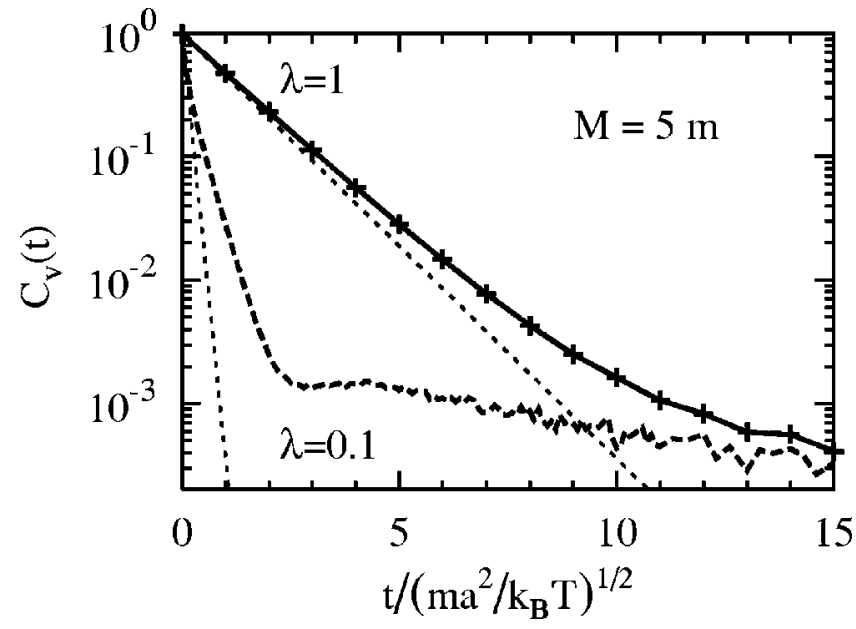

FIG. 9. Time dependence of the normalized velocity autocorrelation function of a heavy particle of mass $M=5 \mathrm{~m}$ for mean free path $\lambda=1$ and $\lambda=0.1$. Dashed lines correspond to the exponential decays in Eq. (24). In both cases the number density is $\rho=5$ and the rotation angle $\alpha=130$. Compare with Fig. 3.

played at lower number density $\rho$, but an asymptotic curve is clearly approached for large values of $\rho$. The deviations for small $\rho$ are due to the presence of density fluctuations.

The dependence of the VACF of a single heavy tracer particle of mass $M=5 m$ on the mean free path $\lambda$ of the solvent is shown in Fig. 9. Corresponding results for the simple fluid are shown in Fig. 3. For $\lambda=0.1$, the qualitative behavior of tracer particles with $M=m$ and $M=5 m$ is very similar. The first collision perfectly follows the molecular-chaos approximation, followed by a slower-than-exponential decay for intermediate times and a crossover to a power-law decay for long times. However, note that since the exponential decay is slower for the heavy particle, the deviations from Brownian behavior appear when the VACF has decayed to approximately one third of its original value for the employed values of $\rho$ and $\alpha$, while for the simple fluid case the VACF has decayed to $6 \%$ of its original value. This implies that the hydrodynamic enhancement is more pronounced for particles of larger mass. For $\lambda=1$, small deviations from the exponential decay are visible for short times; for long times, the crossover to the power-law behavior can be seen.

Analytical approximation for the diffusion coefficient can be calculated similar to Sec. V A. It reads

$$
D_{0}=\frac{k_{B} T}{M} h\left(\frac{1}{\gamma}-\frac{1}{2}\right),
$$

where the decorrelation factor $\gamma$ is now given by Eq. (25).

Simulation results for the diffusion coefficient $D_{M}$ of a heavy tracer particle are plotted in Fig. 10 as a function of the mass $M / m$, for fixed solvent density $\rho=5$ and two different sets of parameters. The agreement of the simulations with the approximation (26) is again very good for parameter values within the particle regime, $\lambda=1$ and $\alpha=45$, but not within the collective regime, $\lambda=0.1$ and $\alpha=130$. This is the same behavior as observed in the simple fluid (see Fig. 7)

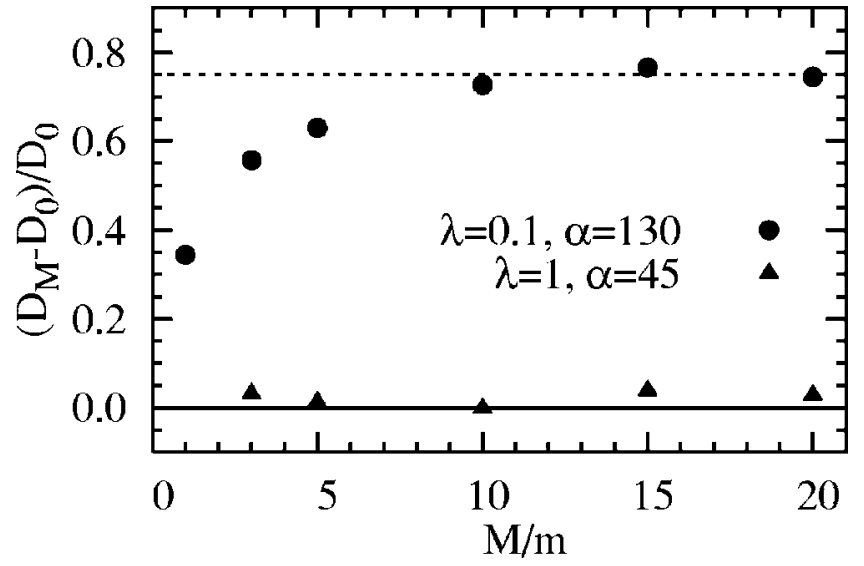

FIG. 10. Relative deviation of the simulated diffusion coefficient $D_{M}$ from the Brownian approximation $D_{0}$, in Eq. (26), as a function of the heavy particle mass for $\rho=5$. These deviations represent the hydrodynamic contribution to the diffusion coefficient $D_{H}=D_{M}-D_{0}$ in units of the Brownian contribution. Symbols are simulation results and the dashed line is a guide to the eye which represents a $75 \%$ enhancement of the hydrodynamic term over the Brownian one.

and indicates again the presence of a hydrodynamic contribution to the diffusion coefficient in the collective regime.

In Fig. 11, the hydrodynamic contribution to the diffusion coefficient (in units of the Brownian contribution) is plotted as a function of the scaled mean free path $\lambda$ for a heavy tracer particle of mass $M=5 \mathrm{~m}$ and for a simple fluid tracer particle (compare Fig. 7). It can be seen that $D_{H}$ increases considerably for small $\lambda$ in both cases. This increment is significantly more pronounced for the heavy particle, which corresponds to the slower decay of the VACF in Fig. 9 for the larger mass. A small deviation of the VACF from the exponential decay was observed in Fig. 9 at short times for $\lambda=1$. This deviation translates into the small hydrodynamic enhancement of the diffusion coefficient of the heavy particle

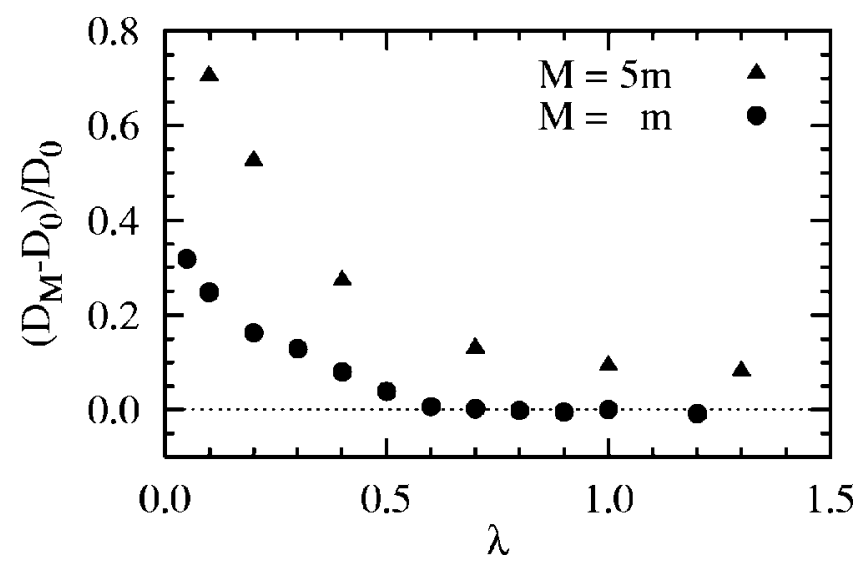

FIG. 11. Hydrodynamic contribution to the diffusion coefficient in units of the Brownian contribution as a function of the scaled mean free path $\lambda$. Symbols are simulation measurements, and the ordinate zero axis represents perfect agreement with the analytical expression $D_{0}$. Simulation parameters are $\alpha=130, \rho=5$, and $L / a$ =20. Compare with Fig. 7 . 
that can be seen in Fig. 11, even at "large" mean free paths $\lambda \simeq 1$.

Figure 10 shows that for a fixed density $\rho$ in the collective regime, the hydrodynamic enhancement increases with increasing mass of the solute particle until $M / m \simeq 2 \rho$, and then levels off and becomes independent of the solute mass for $M / m \gg \rho$. This is consistent with the diffusion behavior of colloidal spheres, where the diffusion coefficient is independent of the mass of the colloidal particles.

Kikuchi et al. [19] determine numerically the friction coefficient acting on a particle of mass $M$ and velocity $\mathbf{v}$ in a MPCD solvent. Their simulation results, for a fluid of $\lambda$ $\simeq 0.9$, compare nicely with the analytical prediction, independently on the mass of the particle. However, we want to point out that this agreement is not very surprising, since their result is obtained from the velocity autocorrelation function after the first collision step, where the molecularchaos approximation is always exact (see Sec. IV C).

The increase of the hydrodynamic coupling of solute and solvent with increasing solute mass can be understood as follows. The relative mass of the solute and solvent particles appears in the collision step via the calculation of the centerof-mass velocity. If solute particles have the same mass as solvent particles and there is a large number of solvent particles per cell, the solvent particles transfer a large random momentum to the solute particle. Simultaneously, the effect of the solute particle momentum on the solvent is small. For this reason, the hydrodynamic contribution to the diffusion constant of a particle of equal mass, shown in Fig. 7, is only of the order of $30 \%$ for the largest Schmidt number considered. In contrast, this hydrodynamic enhancement is $65 \%$ when $M / m \simeq \rho$ and $75 \%$ when $M / m \gtrsim 2 \rho$, as can be seen in Fig. 10. A very large mass of the solute particle is not very convenient either, because it implies a large ballistic regime and a long diffusion time. Therefore, we conclude that a mass $M / m \simeq \rho$ for the solute particle is an optimal choice to enhance the hydrodynamic coupling between solute and fluid particles.

\section{B. Finite concentration of heavy pointlike particles}

At a finite concentration of solute particles, an important question is to which extent solute particles build up hydrodynamic interactions among themselves through the fluid particles when simulated with MPCD. We study therefore systems with different concentrations of heavy particles for sets of parameters within the particle and the collective regimes, respectively. We address this question by investigating the tracer-diffusion coefficient.

Simulations with different heavy particle concentrations are performed by changing the total number $N_{M}$ of heavy particles but keeping fixed the volume $V=L^{3}$ and the number $N$ of solvent particles. The corresponding number density of heavy particles is defined as $\phi=N_{M}(a / L)^{3}$. In Fig. 12, the diffusion coefficients for three different values of the mean free path are displayed. Very surprisingly, when the data are normalized by the corresponding diffusion coefficients in the limit of vanishing density $\phi$, all three data sets, which are both in the particle and the collective regime, collapse onto a

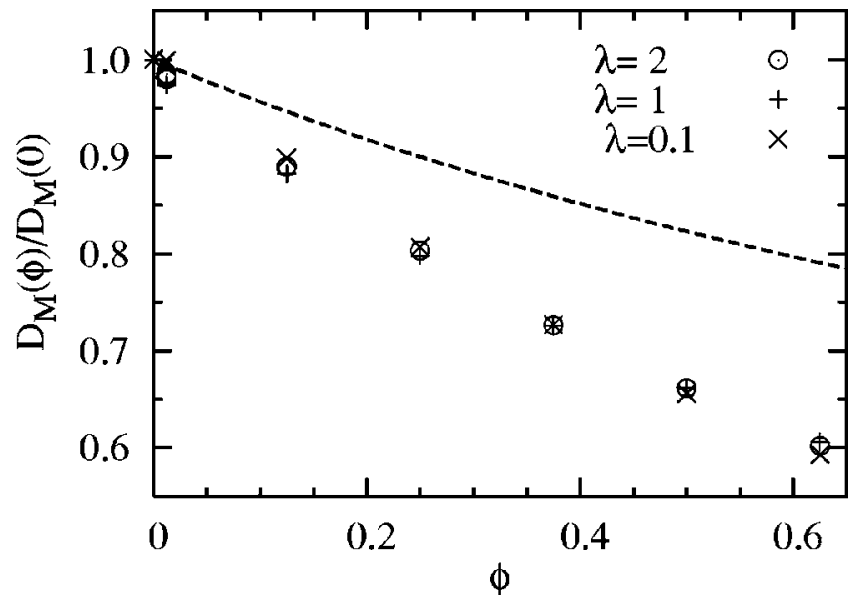

FIG. 12. Diffusion coefficients for a heavy particle as a function of the concentration $\phi=N_{M}(a / L)^{3}$, normalized with the diffusion coefficient $D_{M}(0)$ of heavy particles at zero number density. The dashed line is the analytical approximation from Eqs. (26) and (29), symbols correspond to the simulation data with $\lambda$ specified in the legend. The other parameters are $M / m=\rho=5, \alpha=130$, and $L / a$ $=20$.

single curve. We recall that the hydrodynamic enhancement for the diffusion coefficient of a single heavy particle, here denoted as $D_{M}(0)$, is quite different among these three values of $\lambda$ (see Fig. 11). It can be inferred from the data collapse in Fig. 12 that there is no extra hydrodynamic contribution among these heavy particles, which is consistent with the idea that there is no hydrodynamic screening for point particles $[38,39]$.

The dependence of the diffusion coefficient on the heavy particle number density can be understood along the same lines as for the simple fluid or the single heavy particle. We assume that in each collision box there is a fixed number of fluid particles $\rho$, but that the number of heavy particles $n$, fluctuates from one collision box to another. The probability $P(n)$ of a given heavy particle to be found in a cell with a total of $n-1$ other heavy particles is given by the Poisson distribution function, $P(n)=e^{-\phi} \phi^{n-1} /(n-1)$ !. The corresponding decorrelation factor for a heavy particle in a collision box with $(n-1)$ other heavy particles and $\rho$ fluid ones is

$$
\gamma_{n}=1-M /(\rho m+n M),
$$

compare the definition of $\gamma_{1}$ in Eq. (25) for a single heavy particle in a collision box. The diffusion coefficient is then given by Eq. (26), where the decorrelation factor is now $\gamma$ $=\gamma_{\alpha} \sum_{n=1}^{\infty} P(n) \gamma_{n}$. In the regime of low number density, $\phi$ $\ll 1$, this implies

$$
\gamma=\gamma_{\alpha}\left[(1-\phi) \gamma_{1}+\phi \gamma_{2}+O\left(\phi^{2}\right)\right] .
$$

In the special case of $\rho=M / m$, the sum can be evaluated analytically and yields

$$
\gamma=\gamma_{\alpha}\left[1-\left(e^{-\phi}+\phi-1\right) / \phi^{2}\right] .
$$

In Fig. 12 the simulation data for the normalized diffusion coefficient at different volume fractions are compared with the theoretical prediction obtained from Eq. (26) with the 
decorrelation function in Eq. (29). It can be seen that this prediction overestimates the values for the diffusion coefficients. Further studies are required to understand the origin of this deviation.

\section{HYBRID DYNAMICS}

In order to go one step further in the development of an efficient simulation technique for suspensions of colloidal particles with MPCD, we next investigate the effect of excluded-volume interactions between the heavy particles. To this end, the MPCD algorithm has to be combined with standard molecular dynamics (MD) for the solute particles.

\section{A. The Model}

We consider a dispersion of spherical colloidal particles in three dimensions. The interactions of solvent particles among themselves and with colloids take place in the MPCD collisional step, exactly in the same way as described for the heavy pointlike particles in Sec. VI A. However, the streaming step (1) is used only for the solvent particles. The position update of the colloidal particles is performed in several MD steps between MPCD collisions. In these MD steps, colloids interact via an excluded-volume potential. We use the truncated repulsive Lennard Jones potential [40]

$$
V^{\mathrm{RLJ}}(r)= \begin{cases}4 \varepsilon\left[\left(\frac{\sigma}{r}\right)^{12}-\left(\frac{\sigma}{r}\right)^{6}\right]+\varepsilon, & r \leqslant r_{\min }, \\ 0, & r>r_{\min },\end{cases}
$$

where $r$ is the distance between the centers of the colloidal particles. The parameter $\sigma$ is related to the particle diameter; it is chosen to equal the collision box length, $\sigma=a$, so that there is typically no more than one colloid particle in each collision box. The potential strength is taken to be equal to the thermal energy $\varepsilon=k_{B} T$, the cutoff radius is $r_{\min }=2^{1 / 6} \sigma$, and the mass of the particles is taken to be $M=5 \mathrm{~m}$. The MD time steps are integrated with the velocity-Verlet algorithm [41] with a time step $\Delta t=0.002 \sqrt{\varepsilon / m a^{2}}$.

In other words, we consider a system of colloidal particles interacting through repulsive Lennard Jones potentials whose positions and velocities evolve in discrete time intervals $\Delta t$. This procedure is interrupted every $h / \Delta t$ steps for the interaction with the fluid particles. This interaction is a MPCD event where solvent and solute particles interchange momentum. This implies that the solvent particles can enter the cores of the colloidal particles, but the colloids cannot interpenetrate each other.

The hybrid model described here is a variant of the model introduced previously by Malevanets and Kapral $[15,42]$. In their model, both the solute-solute and solute-solvent interactions were taken into account through excluded-volume potentials with $\mathrm{MD}$, and only the solvent-solvent interactions were mesoscopically described through MPCD. The advantage of the model described here comes from the fact that in the MD steps just the solute particles are considered. This leads to a considerable speed up of the simulations.

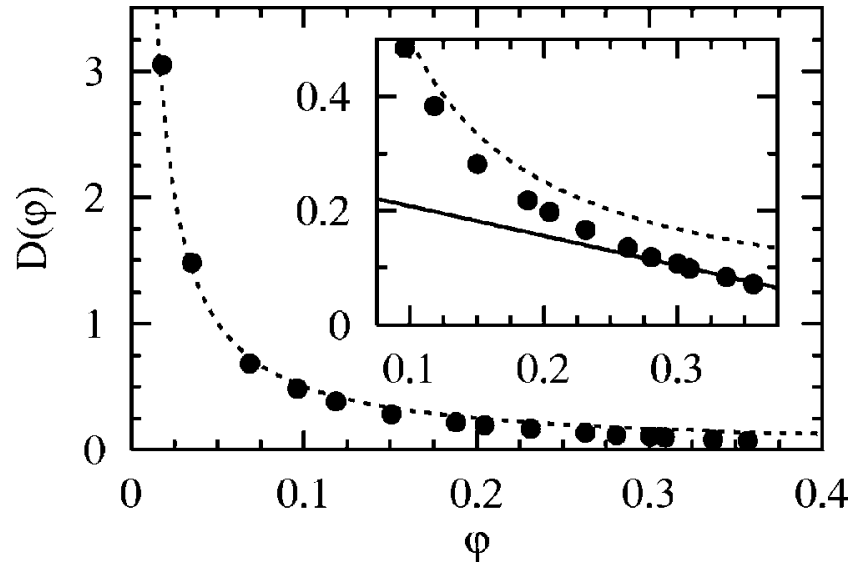

FIG. 13. Diffusion coefficient for colloidal particles without solvent as a function of the volume fraction $\varphi$. Symbols are simulation results, the dashed line corresponds to the analytical prediction (32). The inset is a zoom over the small values of the diffusion coefficient, and the solid line is a linear extrapolation for large values of $\varphi$.

\section{B. Diffusion in colloidal dispersions}

We measure the diffusion coefficient of the dispersion through the mean-square displacement of a tracer particle, as before. Simulations are performed for different colloidal concentrations. The volume fraction of colloidal particles $\varphi$ is the fraction of the total volume $V$ occupied by the colloidal particles $\varphi=(\pi / 6) \sigma_{\text {eff }}^{3} \rho_{M}$, where the effective diameter $\sigma_{\text {eff }}$ is determined by the Barker-Henderson expression [43]

$$
\sigma_{\text {eff }}=\int_{0}^{r_{\min }} d r\left[1-\exp \left(-V^{\mathrm{RLJ}}(r) / k_{B} T\right)\right]
$$

For our choice of Lennard-Jones parameters, this gives $\sigma_{\text {eff }}$ $=1.01 \sigma$. The number density of colloidal particles is $\rho_{M}$ $=\left(N_{M}-1\right) / V \simeq N_{M} / V$, where $N_{M}$ is the number of heavy particles with excluded-volume interactions.

For later comparison and better understanding of our hybrid model results, we recall first the basic behavior of a system with excluded-volume interactions only. In Fig. 13 we show the results for the diffusion coefficient of a MD simulation of repulsive Lennard-Jones particles. Kinetic theory for hard spheres predicts in the low-density limit [44]

$$
D_{\mathrm{MD}}(\varphi)=\frac{3}{8 \sigma_{\text {eff }}^{2}} \sqrt{\frac{k_{B} T}{\pi M}} \frac{1}{\rho_{M}} .
$$

This analytical prediction is depicted in Fig. 13 together with the simulation results. It can be seen that for small volume fraction, the $\varphi^{-1}$ behavior is properly reproduced, while for large volume fractions a linear behavior can be inferred.

The density dependence of the self-diffusion coefficient of colloidal hard spheres in a hydrodynamic bath can be found in Ref. [20],

$$
D_{S}(\varphi)=D_{S}(0)\left[1-2.1 \varphi+O\left(\varphi^{2}\right)\right] .
$$

The diffusion coefficient now decreases linearly with the volume fraction, in contrast with the kinetic theory result (32) 


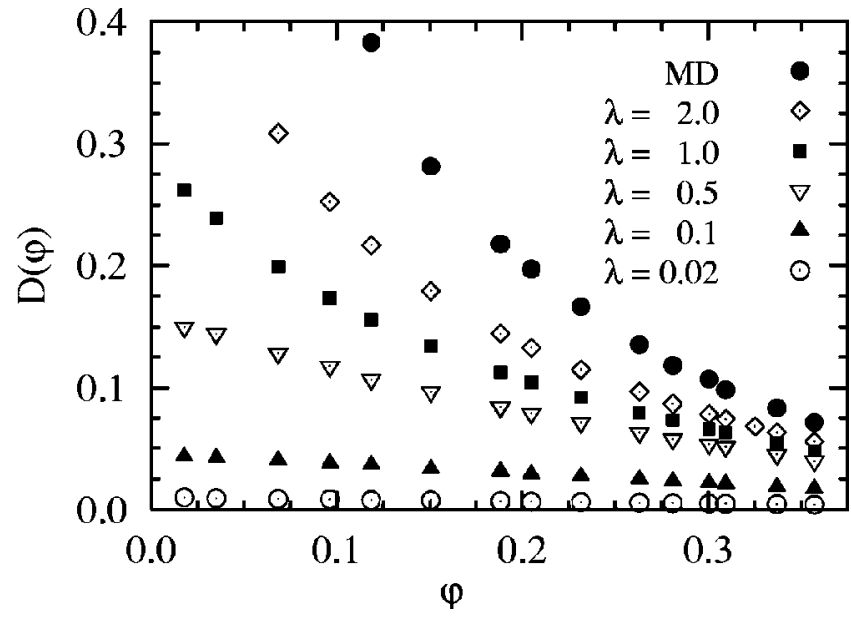

FIG. 14. Diffusion coefficient as a function of the volume fraction of colloidal particles dispersions interacting with a solvent represented with MPCD at different collision times. For comparison, the MD results of Fig. 13 are also plotted.

for a gas of hard spheres. In the calculation of Eq. (33), Brownian and hydrodynamic terms have to be considered, and it has been found that the hydrodynamic terms almost cancel. For a colloidal dispersion in a Brownian bath [20] the first-order correction in Eq. (33) equals $-2.0 \varphi$. Thus, no significant differences are expected between Brownian and hydrodynamic measurements of the diffusion coefficient.

Simulation results with the hybrid method are shown in Fig. 14. The simulations presented here are performed with rotation angle $\alpha=130$, fluid number density $\rho=5$, and mass $M=5 m$ of the colloidal particle. We vary the mean free path between $\lambda=0.02$ and $\lambda=2.0$.

In the limit of very small volume fractions, the repulsive interactions between colloids are negligible, and the colloidal dispersion will behave as the dispersion of heavy pointlike particles presented in Secs. VI A and VI B. In this limit, we know from Eq. (26) that the diffusion coefficient $D(0)$ increases with the mean free path $\lambda$ [with $D(0) \sim \lambda$ in the molecular-chaos approximation]. The decrease of $D(\varphi)$ with decreasing $\lambda$ displayed in Fig. 14 arises then as a natural consequence. Furthermore, the MPCD interactions of the colloid particles with the fluid imply that the self-diffusion coefficient at small densities does not diverge but goes to finite value dictated by Eq. (26).

For small but finite volume fractions, in the case of large values of $\lambda$, we observe a behavior reminiscent of the $\varphi^{-1}$ decay of hard-sphere gases, instead of the linear decrease expected from Eq. (33). This can be understood since, in the limit of very large mean free paths, the colloids will essentially interact with each other rather than with the solvent. This behavior is not seen in experiments of colloidal dispersions, because the diffusive length scale is typically much smaller than the diameter of the particles.

Therefore, the appropriate parameters for the modeling of colloidal dispersions have again to be chosen in the collective regime. In Fig. 15, the normalized diffusion coefficient is shown, where $D(0)$ is extrapolated from the simulated data. The linear behavior in Eq. (33) is indeed observed for

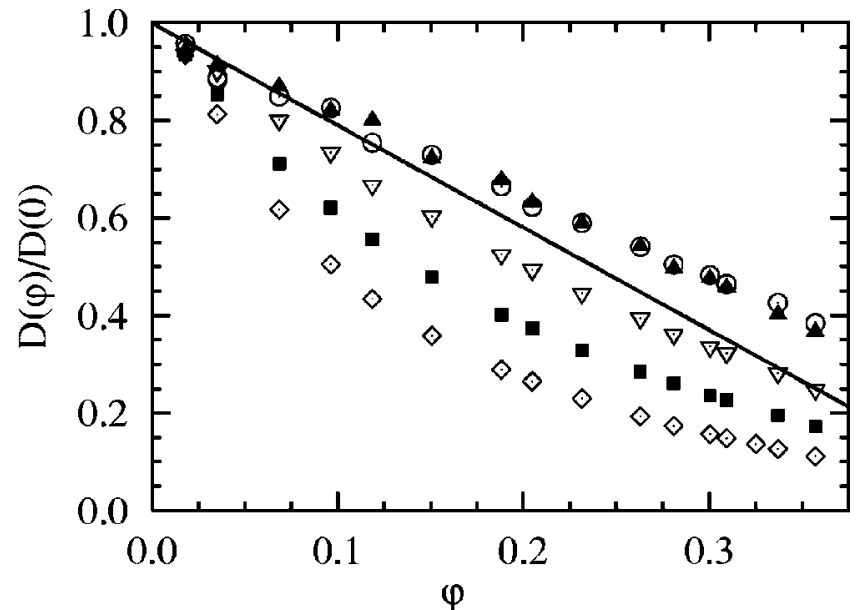

FIG. 15. Dependence of the normalized diffusion coefficient on the volume fraction $\varphi$ of colloidal particles. The same data are shown as in Fig. 14. The normalization factor $D(0)$ is obtained by extrapolation of the data to zero volume fraction. The solid line corresponds to the hydrodynamic prediction in Eq. (33).

the smallest values of the mean free path $\lambda=0.02$ and $\lambda$ $=0.1$, within the accuracy of the simulations. Thus, we find that in order to obtain the theoretically predicted behavior (33) from simulation of the MD-MPCD hybrid model, small values of the mean free path and large values of the rotation angle $\alpha$ are required, i.e., parameters in the collective regime.

However, an almost identical dependence of the diffusion coefficient on the volume fraction is predicted theoretically in the absence of hydrodynamics interactions. In order to investigate this point in more detail, we have performed simulations of a hybrid model similar to the one presented here, but with a completely Brownian solvent. One way of transforming a MPCD fluid in a Brownian solvent has been introduced by Kikuchi et al. [45], where the velocities among all the fluid particles are randomly interchanged after each MPCD collision step. We propose an alternative method which does not consider any solvent particles. Instead, at every $h / \Delta t$ steps the MD dynamics is interrupted for a rotation of the (full) velocity of each colloid around a random axis by and angle $\alpha$. In this case, the diffusion coefficient at zero volume fraction $D(0)$ is given by Eq. (26) but the decorrelation factor is $\gamma \equiv \gamma_{\alpha}$ with $\gamma_{\alpha}$ of Eq. (6). The simulation results of the dependence of the diffusion coefficient on the volume fraction are quite similar to those displayed in Fig. 15. The data for $D(\varphi)$ follow a linear decay only for very small values of $\lambda$, where the friction is large and $D(0)$ is small enough to represent a fluid. For large values of $\lambda, D(\varphi)$ has a concave shape, reminiscent of the $\varphi^{-1}$ behavior of gases, similarly as observed for the hydrodynamic simulations.

Simulations with a similar hybrid method of a twodimensional colloidal suspension have been reported by Falck et al. [33]. In the majority of the presented results, they consider excluded-volume interaction among colloids but not between colloids and solvent particles. They measure an apparent tracer diffusion coefficient (since the diffusion coeffi- 
cient in two dimensions diverges with increasing system size) of the colloids for different concentrations. Three different Schmidt numbers are studied. A similar trend in the data is observed as in our simulations: the normalized diffusion coefficient increases with increasing Schmidt number, in particular for intermediate values of the volume fraction $\varphi$. However, our interpretation is different. While Falck et al. attribute this effect to hydrodynamics, we believe that it is due to the crossover from gaslike to diffusive behavior of the colloidal dynamics.

In summary, our hybrid model describes the dynamics of a dispersion of hard-sphere colloids very well in the collective regime of the solvent. In the hydrodynamic interaction, only the leading contribution for large distances is included in our model. This implies that lubrication forces between neighboring particles at short distances, as well as the coupling between rotational degrees of freedom, are neglected. We conclude from the very weak dependence of our results for the normalized diffusion coefficients on the mean free path, which controls the strength of the hydrodynamic interaction, that our model works very well for not too concentrated colloidal dispersions.

\section{SUMMARY AND CONCLUSIONS}

In this paper we have performed a detailed analysis of the hydrodynamic properties of a fluid simulated with MPCD. We identify two hydrodynamic regimes in terms of the parameters of the MPCD algorithm. The particle regime is characterized by dynamical properties being closer to those of a gas than to those of a liquid. The Schmidt number is small and the dominant transport mechanism is kinetic transport. This is the regime obtained for large values of the collision time and/or small values of the rotation angle. The second and more relevant regime for fluid simulations is the collective regime. In this regime the Schmidt number is large and collisional transport dominates over kinetic transportthis characterizes liquidlike behavior. These properties are obtained for large values of the rotation angle and small values of the collision time.

Different quantities have been measured in both regimes. The main conclusion is that the diffusion coefficient shows a hydrodynamic enhancement in the collective regime. In the study of the VACF we observe that the behavior can be understood in both regimes as an exponentially decay for short times and algebraic decay for long times. In the particle regime, a simple crossover between both behaviors is observed while an extra intermediate behavior is displayed in the col- lective regime. This intermediate behavior of the VACF is typically a slower than the initial exponential decay. We have shown that the origin of this intermediate decay region is due to the build-up of correlations by many-body collisions, which is in conceptual agreement with the hydrodynamic behavior. The theoretical predictions for the diffusion coefficient are based on a molecular-chaos assumption, which gives an exponential decay of the VACF. Consequently, a deviation from the theoretical prediction is found in the collective regime. This deviation can be understood as a hydrodynamic contribution to the Brownian value.

In a further step, we have investigated the differences between the particle and the collective regime for complex fluids. We have studied the behavior of heavy particles embedded in the MPCD fluid which can represent solute or colloidal particles dissolved in a simple fluid. This study demonstrates that optimal hydrodynamic coupling occurs when the mass of the tagged particle is on the order of the solvent mass in a collision cell.

In order to describe colloidal dispersions at finite volume fractions, it is necessary to account for excluded volume interactions among colloidal particles. To this end, a hybrid model was studied, which combines MPCD for the solvent with MD simulations for the colloidal particles. We show that only for parameters within the collective regime does the hybrid model reproduce the proper hydrodynamic behavior. In this case, the results agree well with the theoretical calculations with and without hydrodynamic interactions, as well as with experimental results.

A more precise modeling of colloidal particles would require new interactions among fluid and colloids such that fluid particles would not freely travel through colloidal particles and eventually angular momentum could be interchanged among them. In the future, it will be interesting to explore in which applications a more detailed description of colloidal interactions is necessary, as compared to our simplified model which allows more particles and larger system sizes, and is therefore well suited to study cooperative phenomena.

\section{ACKNOWLEDGMENTS}

We thank G. Vliegenthart, H. Noguchi, D. M. Kroll, T. Ihle, N. Kikuchi, and A. Lamura for helpful discussions. Financial support of this work by the German Research Foundation (DFG) within the SFB TR6, "Physics of Colloidal Dispersions in External Fields," is gratefully acknowledged. M.R. also acknowledges partial support from the Project Nos. BFM2001-0290 and FIS2004-01934.
[1] U. Frisch, B. Hasslacher, and Y. Pomeau, Phys. Rev. Lett. 56, 1505 (1986).

[2] J. M. V. A. Koelman, Phys. Rev. Lett. 64, 1915 (1990).

[3] G. R. McNamara and G. Zanetti, Phys. Rev. Lett. 61, 2332 (1988).

[4] P. Lallemand and L. S. Luo, Phys. Rev. E 61, 6546 (2000).
[5] S. Succi, The Lattice Boltzmann Equation: For Fluid Dynamics and Beyond (Clarendon, Oxford, 2001).

[6] R. Gingold and J. Monaghan, Mon. Not. R. Astron. Soc. 181, 375 (1977).

[7] O. Kum, W. G. Hoover, and H. A. Posch, Phys. Rev. E 52, 4899 (1995). 
[8] P. J. Hoogerbrugge and J. M. V. A. Koelman, Europhys. Lett. 19, 155 (1992).

[9] P. Español and P. Warren, Europhys. Lett. 30, 191 (1995).

[10] R. D. Groot and P. B. Warren, J. Chem. Phys. 107, 4423 (1997).

[11] N. Metropolis, A. W. Rosenbluth, M. N. Rosenbluth, A. H. Teller, and E. Teller, J. Chem. Phys. 21, 1087 (1953).

[12] G. A. Bird, Molecular Gas Dynamics (Clarendon, Oxford, 1976).

[13] H. Tanaka and T. Araki, Phys. Rev. Lett. 85, 1338 (2000).

[14] A. Malevanets and R. Kapral, J. Chem. Phys. 110, 8605 (1999).

[15] A. Malevanets and R. Kapral, J. Chem. Phys. 112, 7260 (2000).

[16] T. Ihle and D. M. Kroll, Phys. Rev. E 67, 066705 (2003).

[17] T. Ihle and D. M. Kroll, Phys. Rev. E 67, 066706 (2003).

[18] E. Tüzel, M. Strauss, T. Ihle, and D. M. Kroll, Phys. Rev. E 68, 036701 (2003).

[19] N. Kikuchi, C. M. Pooley, J. F. Ryder, and J. M. Yeomans, J. Chem. Phys. 119, 6388 (2003).

[20] J. K. G. Dhont, An Introduction to Dynamics of Colloids (Elsevier, Amsterdam, 1996).

[21] E. Allahyarov and G. Gompper, Phys. Rev. E 66, 036702 (2002).

[22] T. Ihle and D. M. Kroll, Phys. Rev. E 63, 020201(R) (2001).

[23] T. Ihle, E. Tüzel, and D. M. Kroll, Phys. Rev. E 70, 035701(R) (2004).

[24] A. Lamura, G. Gompper, T. Ihle, and D. M. Kroll, Europhys. Lett. 56, 319 (2001).

[25] D. J. Tritton, Physical Fluid Dynamics 2nd ed. (Oxford Science Publications, Oxford, 1988).

[26] I. Pagonabarraga, M. H. J. Hagen, and D. Frenkel, Europhys. Lett. 42, 377 (1998).

[27] M. Ripoll, M. H. Ernst, and P. Español, J. Chem. Phys. 115,
7271 (2001).

[28] B. Alder and T. Wainwright, Phys. Rev. A 1, 18 (1970).

[29] M. H. Ernst, E. H. Hauge, and J. M. J. van Leuwen, Phys. Rev. A 4, 2055 (1971).

[30] M. H. Ernst, Phys. Rev. E 71, 030101(R) (2005).

[31] M. Ripoll and M. H. Ernst, e-print cond-mat/0502331, Phys. Rev. E (to be published).

[32] K. Tucci and R. Kapral, J. Chem. Phys. 120, 8262 (2004).

[33] E. Falck, O. Punkkinen, I. Vattulainen, and T. Ala-Nissila, Eur. Phys. J.: Appl. Phys. 13, 267 (2004).

[34] M. Ripoll, K. Mussawisade, R. G. Winkler, and G. Gompper, Europhys. Lett. 68, 106 (2004).

[35] A. Malevanets and J. M. Yeomans, Europhys. Lett. 52, 231 (2000).

[36] R. G. Winkler, K. Mussawisade, M. Ripoll, and G. Gompper, J. Phys.: Condens. Matter 16, S3941 (2004).

[37] I. Ali, D. Marenduzzo, and J. M. Yeomans, J. Chem. Phys. 121, 8635 (2004).

[38] P. Ahlrichs, R. Everaers, and B. Dünweg, Phys. Rev. E 64, 040501(R). (2001).

[39] A. J. C. Ladd, Phys. Rev. Lett. 76, 1392 (1996).

[40] H. C. Andersen, D. Chandler, and J. D. Weeks, Adv. Chem. Phys. 34, 105 (1976).

[41] M. P. Allen and D. J. Tildesley, Computer Simulations in Liquids (Clarendon, Oxford, 1987).

[42] A. Malevanets and R. Kapral, Lect. Notes Phys. 640, 116 (2004).

[43] J. A. Barker and D. Henderson, Rev. Mod. Phys. 48, 587 (1976).

[44] J. P. Boon and S. Yip, Molecular Hydrodynamics (McGrawHill, New York, 1980).

[45] N. Kikuchi, A. Gent, and J. M. Yeomans, Eur. Phys. J.: Appl. Phys. 9, 63 (2002). 